\title{
Cerebrovascular reactivity to hypercapnia during sevoflurane or desflurane anesthesia in rats
}

\author{
Koji Sakata, Kazuhiro Kito, Naokazu Fukuoka, Kiyoshi Nagase, \\ Kumiko Tanabe, and Hiroki Iida \\ Department of Anesthesiology and Pain Medicine, Gifu University Graduate School of Medicine, Gifu, Japan
}

\begin{abstract}
Background: Hypercapnia causes dilation of cerebral vessels and increases cerebral blood flow, resulting in increased intracranial pressure. Sevoflurane is reported to preserve cerebrovascular carbon dioxide reactivity. However, the contribution of inhaled anesthetics to vasodilatory responses to hypercapnia has not been clarified. Moreover, the cerebrovascular response to desflurane under hypercapnia has not been reported. We examined the effects of sevoflurane and desflurane on vasodilatory responses to hypercapnia in rats.

Methods: A closed cranial window preparation was used to measure the changes in pial vessel diameters. To evaluate the cerebrovascular response to hypercapnia and/or inhaled anesthetics, the pial vessel diameters were measured in the following states: without inhaled anesthetics at normocapnia (control values) and hypercapnia, with inhaled end-tidal minimal alveolar concentration (MAC) of 0.5 or 1.0 of either sevoflurane or desflurane at normocapnia, and an MAC of 1.0 of sevoflurane or desflurane at hypercapnia.

Results: Under normocapnia, 1.0 MAC, but not 0.5 MAC, of sevoflurane or desflurane dilated the pial arterioles and venules. In addition, under both 1.0 MAC of sevoflurane and 1.0 MAC of desflurane, hypercapnia significantly dilated the pial arterioles and venules in comparison to their diameters without inhaled anesthetics. The degrees of vasodilation were similar for desflurane and sevoflurane under both normocapnia and hypercapnia.

Conclusions: Desflurane induces cerebrovascular responses similar to those of sevoflurane. Desflurane can be used as safely as sevoflurane in neurosurgical anesthesia.
\end{abstract}

Keywords: Cerebrovascular circulation; Desflurane; Hypercapnia; Sevoflurane.

Corresponding author: Hiroki Iida, M.D., Ph.D.

Department of Anesthesiology and Pain Medicine, Gifu University Graduate School of Medicine, 1-1 Yanagido, Gifu 501-1194, Japan

Tel: 81-58-230-6404, Fax: 81-58-230-6405

Email: iida@gifu-u.ac.jp

ORCID: https://orcid.org/0000-0003-0924-598X

It was presented at ANESTHESIOLOGY 2016 Annual Meeting of The American Society of Anesthesiologists (ASA), October 2016, Hyatt Regency McCormick Place, Chicago, USA.

Received: August 21, 2018.

Revised: November 6, 2018.

Accepted: November 26, 2018.

Korean J Anesthesiol 2019 June 72(3): 260-264

https://doi.org/10.4097/kja.d.18.00244

\section{Introduction}

Cerebral blood flow (CBF) is affected by the partial pressure of arterial carbon dioxide $\left(\mathrm{PaCO}_{2}\right)$ [1]. Desflurane may offer advantages over sevoflurane for early emergence from anesthesia $[2,3]$. The degree of increase in CBF in normocapnia varies according to the concentrations of the volatile anesthetics. The vasodilatory effects are reported to be greater for desflurane or isoflurane than for sevoflurane in normocapnia [4-6]. Hypercapnia dilates cerebral vessels and increases $\mathrm{CBF}$, resulting in elevation of intracranial pressure [7]. Therefore, it is important to investigate the effect of volatile anesthetics on CBF under hypercapnia. Although transcranial Doppler studies indicate that sevoflurane does not alter the vasodilatory effect of hypercapnia

(c) This is an open-access article distributed under the terms of the Creative Commons Attribution Non-Commercial License (http://creativecommons.org/ licenses/by-nc/4.0/), which permits unrestricted non-commercial use, distribution, and reproduction in any medium, provided the original work is properly cited. 
on human cerebral vessels [8-10], there have been no experiments in which cerebral vessels were observed directly. With regard to desflurane, the effects on the cerebrovasculature under hypercapnia have not yet been adequately reported. We investigated the effects of desflurane on the cerebral vascular responses induced by hypercapnia in rats and compared the effects of desflurane and sevoflurane.

\section{Materials and Methods}

The experimental protocol was approved by the Institutional Committee for Animal Care of Gifu University Graduate School of Medicine. The experiments were performed in 14 male Sprague-Dawley rats weighting 300-400 g. Each rat was anesthetized (pentobarbital sodium, $50 \mathrm{mg} / \mathrm{kg}$ body weight, intraperitoneal injection) and then mechanically ventilated (around $60 / \mathrm{min}$ ) through a tracheostomy tube by means of a ventilator (Model 683; Harvard Apparatus, USA) using room air supplemented with oxygen. The inhaled oxygen concentration was $50 \%$. Tidal volume was adjusted to maintain $\mathrm{PaCO}_{2}$ between 35 and $40 \mathrm{mmHg}$ at the beginning of each experiment. Supplementary pentobarbital was administered intravenously by continuous infusion at $4 \mathrm{mg} / \mathrm{kg} / \mathrm{h}$. One femoral artery was cannulated to monitor arterial blood pressure and to provide blood samples. A femoral vein was cannulated for administration of fluid and drugs. Rectal temperature was maintained at $37-38^{\circ} \mathrm{C}$ using a heating pad.

A closed cranial window was used for observation of the pial microcirculation, while the rat's head was fixed in the sphinx position. The scalp was retracted, a hole $3 \times 2 \mathrm{~mm}$ in diameter was made in the bone over the right parietal cortex, and the dura was opened carefully. A polypropylene ring with a fitted glass coverslip was placed over the hole and secured with dental acrylic. The space under the window was filled with artificial cerebrospinal fluid (aCSF). Two catheters were inserted into the ring: one was attached to a reservoir bottle containing aCSF to maintain a constant intracranial pressure; the second was used for draining the fluid. The temperature within the window, which was monitored using a thermistor (Model 6510; Mallinckrodt Medical, USA), was maintained at $37-38^{\circ} \mathrm{C}$. The composition of the aCSF was as follows: $\mathrm{Na}^{+}, 157 \mathrm{mM} / \mathrm{L} ; \mathrm{K}^{+}, 3 \mathrm{mM} / \mathrm{L} ; \mathrm{Ca}^{2+}, 1.5 \mathrm{mM} / \mathrm{L}$; $\mathrm{Mg}^{2+}, 0.7 \mathrm{mM} / \mathrm{L} ; \mathrm{Cl}^{-}, 139 \mathrm{mM} / \mathrm{L} ; \mathrm{HCO}^{3-}, 25 \mathrm{mM} / \mathrm{L}$; urea, 40 $\mathrm{mg} / \mathrm{dl}$; and glucose, $67 \mathrm{mg} / \mathrm{dl}$.

The pial images obtained in these experiments were stored on disk (with time records) for later playback and analysis. For each rat, the diameters of three pial arterioles (baseline diameters, $30-80 \mu \mathrm{m}$ ) and three pial venules (baseline diameters, $30-100 \mu \mathrm{m}$ ) were measured using a videomicrometer (Olympus Flovel videomicrometer, Model VM-20; Flovel, Japan) on a video monitor receiving signals from a microscope (Model SHZ-
10; Olympus, Japan). To minimize bias, we selected the vessels from which data were to be collected at the beginning of the experiment. The percentage changes in pial vessel diameter were used in the statistical analysis. Mean arterial blood pressure and heart rate were continuously monitored.

For measuring pial vessel diameters, first, baseline pial vessel diameters were recorded at normocapnia $(35-40 \mathrm{mmHg})$ under pentobarbital anesthesia. Next, $\mathrm{CO}_{2}$ was added to the respiratory circuit to induce hypercapnia $(55-60 \mathrm{mmHg})$. Five minutes after a steady state of hypercapnia was reached, pial vessel diameters were recorded. $\mathrm{CO}_{2}$ inhalation was then stopped, allowing a return to normocapnia. Five minutes after a steady state at normocapnia was reached, pial vessel diameters were recorded and checked for return to baseline diameters $(n=14)$. Next, we examined the vasodilatory effects of sevoflurane $(n=7)$ or desflurane $(n=7)$ on the responses of the pial vessels to hypercapnia. We recorded the pial vessel diameters in the following states: under inhaled end-tidal minimal alveolar concentration (MAC) of 0.5 or 1.0 for either sevoflurane (2.4 vol\%) [6] or desflurane (5.7 vol\%) [11] at normocapnia, and under an MAC of 1.0 for sevoflurane or desflurane at hypercapnia. Expiratory concentrations of inhalation anesthetics were monitored. Under each state, we measured the vessel diameters 20 minutes after stabilizing the end-tidal concentrations of inhalation anesthetics.

Changes in all variables were examined by one-way analysis of variance (ANOVA) for repeated measurements, with Tukey's test used for post-hoc comparisons. The group differences between sevoflurane and desflurane were compared by two-way ANOVA. Significance was set at $\mathrm{P}<0.05$. All values are presented as mean \pm standard deviation (SD).

\section{Results}

The mean arterial blood pressure, heart rate, arterial blood gas tension, and serum electrolyte levels showed no significant changes throughout the experiments. In both groups, during hypercapnia, $\mathrm{pH}$ was significantly lower than that during normocapnia (Tables 1 and 2). In the sevoflurane group, pial arteriolar and venular diameters under hypercapnia without sevoflurane significantly increased by $9.7 \% \pm 1.5 \%$ and $8.0 \%$ $\pm 1.3 \%$, respectively (Figs. $1 \mathrm{~A}$ and $1 \mathrm{~B}$ ). Under normocapnia with $0.5 \mathrm{MAC}$ of sevoflurane, there were no differences in pial arteriolar and venular diameters compared to the baseline diameters; however, with 1.0 MAC of sevoflurane, pial arteriolar and venular diameters significantly increased by $5.8 \% \pm 2.0 \%$ and $5.6 \% \pm 1.7 \%$, respectively (Figs. $1 \mathrm{~A}$ and $1 \mathrm{~B}$ ). Under hypercapnia with 1.0 MAC sevoflurane, pial arteriolar and venular diameters significantly increased by $13.7 \% \pm 2.6 \%$ and $11.8 \% \pm$ $1.9 \%$, respectively, compared to the baseline diameters. These values were significantly larger than the diameters under hy- 
Table 1. Changes in Hemodynamic and Physiological Parameters during the Experiments in the Sevoflurane Group

\begin{tabular}{|c|c|c|c|c|c|c|c|}
\hline & $\begin{array}{c}\mathrm{HR} \\
\text { (beats/min) }\end{array}$ & $\begin{array}{c}\mathrm{MABP} \\
(\mathrm{mmHg})\end{array}$ & $\mathrm{pH}$ & $\begin{array}{c}\mathrm{PaCO}_{2} \\
(\mathrm{mmHg})\end{array}$ & $\begin{array}{c}\mathrm{PaO}_{2} \\
(\mathrm{mmHg})\end{array}$ & $\begin{array}{c}\mathrm{Na}^{+} \\
(\mathrm{mM} / \mathrm{L})\end{array}$ & $\begin{array}{c}\mathrm{K}^{+} \\
(\mathrm{mM} / \mathrm{L})\end{array}$ \\
\hline Normocapnia (Pre-hypercapnia) & $341 \pm 21$ & $131 \pm 5$ & $7.39 \pm 0.03$ & $37.6 \pm 1.4$ & $229 \pm 12$ & $148 \pm 1$ & $3.4 \pm 0.3$ \\
\hline Hypercapnia & $340 \pm 17$ & $129 \pm 9$ & $7.29 \pm 0.04^{*}$ & $56.6 \pm 1.5^{*}$ & $231 \pm 15$ & $147 \pm 3$ & $3.7 \pm 0.3$ \\
\hline Normocapnia (Post-hypercapnia) & $335 \pm 18$ & $131 \pm 10$ & $7.40 \pm 0.08$ & $38.1 \pm 1.4$ & $231 \pm 11$ & $148 \pm 3$ & $3.8 \pm 0.4$ \\
\hline Normocapnia + 0.5 MAC sevoflurane & $339 \pm 19$ & $129 \pm 10$ & $7.36 \pm 0.02$ & $37.7 \pm 1.7$ & $220 \pm 4$ & $147 \pm 2$ & $4.4 \pm 0.5$ \\
\hline Normocapnia + 1.0 MAC sevoflurane & $341 \pm 19$ & $127 \pm 7$ & $7.40 \pm 0.02$ & $38.3 \pm 1.6$ & $228 \pm 10$ & $146 \pm 2$ & $4.3 \pm 0.4$ \\
\hline Hypercapnia + 1.0 MAC sevoflurane & $344 \pm 19$ & $125 \pm 8$ & $7.23 \pm 0.01^{*}$ & $57.0 \pm 1.7^{*}$ & $232 \pm 18$ & $145 \pm 2$ & $4.2 \pm 0.3$ \\
\hline
\end{tabular}

Values are presented as mean \pm SD. HR: heart rate, MABP: mean arterial blood pressure, MAC: minimum alveolar concentration. ${ }^{*} \mathrm{P}<0.05$ vs. normocapnia.

Table 2. Changes in Hemodynamic and Physiological Parameters during the Experiments in the Desflurane Group

\begin{tabular}{lcccccc}
\hline & $\begin{array}{c}\mathrm{HR} \\
\text { (beats/min) }\end{array}$ & $\begin{array}{c}\mathrm{MABP} \\
(\mathrm{mmHg})\end{array}$ & $\mathrm{pH}$ & $\begin{array}{c}\mathrm{PaCO}_{2} \\
(\mathrm{mmHg})\end{array}$ & $\begin{array}{c}\mathrm{PaO}_{2} \\
(\mathrm{mmHg})\end{array}$ & $\begin{array}{c}\mathrm{Na}^{+} \\
(\mathrm{mM} / \mathrm{L})\end{array}$ \\
\hline Normocapnia (Pre-hypercapnia) & $329 \pm 19$ & $133 \pm 9$ & $7.40 \pm 0.02$ & $36.7 \pm 1.6$ & $209 \pm 36$ & $145 \pm 6$ \\
$(\mathrm{mM} / \mathrm{L})$
\end{tabular}

Values are presented as mean \pm SD. HR: heart rate, MABP: mean arterial blood pressure, MAC: minimum alveolar anesthetic concentration. ${ }^{*} \mathrm{P}<0.05$ vs. normocapnia.

percapnia without sevoflurane or under normocapnia with 1.0 MAC of sevoflurane (Figs. 1A and 1B). In the desflurane group, pial arteriolar and venular diameters under hypercapnia without desflurane significantly increased by $10.0 \% \pm 2.7 \%$ and $6.8 \% \pm$ $2.1 \%$, respectively (Figs. $1 \mathrm{C}$ and $1 \mathrm{D}$ ). Under normocapnia with 0.5 MAC of desflurane, there were no differences in pial arteriolar and venular diameters compared to the baseline diameters. However, with 1.0 MAC of desflurane under normocapnia, pial arteriolar and venular diameters significantly increased by $6.1 \% \pm 2.8 \%$ and $4.1 \% \pm 2.1 \%$, respectively (Figs. $1 \mathrm{C}$ and $1 \mathrm{D}$ ). Under hypercapnia with 1.0 MAC desflurane, pial arteriolar and venular diameters significantly increased by $14.9 \% \pm 2.8 \%$ and $11.3 \% \pm 1.6 \%$, respectively, compared to the baseline diameters. These values were significantly higher than the diameters under hypercapnia without desflurane or under normocapnia with 1.0 MAC of desflurane (Figs. 1C and 1D). There were no differences between the vasodilatory effects in the desflurane and sevoflurane groups. The vasodilation responses to hypercapnia with or without inhaled anesthetics did not significantly differ between cerebral arterioles and venules.

\section{Discussion}

In the present study, we confirmed that volatile anesthetics such as sevoflurane or desflurane dilated pial vessels in a concentration-dependent manner and enhanced hypercapnia-in- duced cerebrovascular dilation. The rates of vasodilation were similar for sevoflurane and desflurane at 1.0 MAC under both normocapnia and hypercapnia.

Both desflurane and sevoflurane increase CBF in a dose-dependent manner [4-6,12-15]. Volatile anesthetics increase CBF and intracranial pressure via their vasodilatory effects [7]. In normocapnia, the degree of increase in CBF varies according to the concentration of each volatile anesthetic [4-6]. The vasodilatory effects of desflurane and isoflurane are greater than those of sevoflurane [4-6]. In these previous studies, CBF was compared between 2.0 MAC isoflurane and sevoflurane [4] or between 1.5 MAC isoflurane and desflurane [5]. However, our study showed no difference between the vasodilatory effects of 1.0 MAC sevoflurane and desflurane on the cerebral pial vessels under normocapnia. We suggest that inhaled anesthetics may induce different increases in CBF only at high concentrations. We have previously reported that activation of adenosine triphosphate-sensitive $\mathrm{K}^{+}$channels is involved in vasodilation of rat cerebral vessels by isoflurane or sevoflurane [12]. However, the mechanism underlying the vasodilatory effects of volatile anesthetics is not entirely clear.

Some studies have reported that the cortical vein pressure is closely correlated with the intracranial pressure and that an increase in cerebral vein pressure causes an increase in the intracranial pressure $[16,17]$. Therefore, compared to arteriole vasodilation, venule vasodilation might contribute to increases 

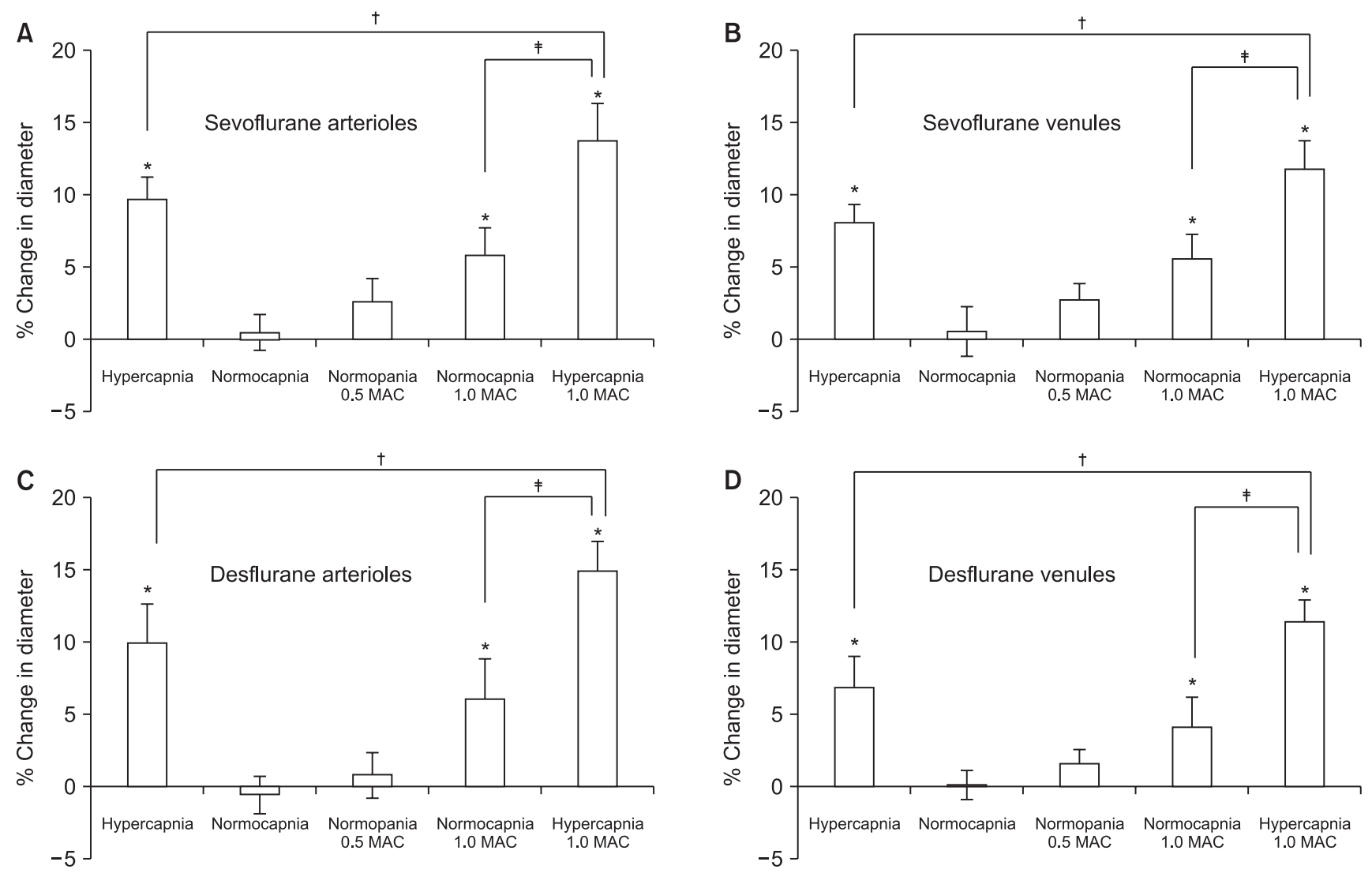

Fig. 1. Effect of sevoflurane (A, B) or desflurane (C, D) on pial arteriolar (A, C) and venular (B, D) diameter under pentobarbital anesthesia. There was no significant difference in the extent of vasodilation between the sevoflurane and desflurane groups. Values are presented as mean \pm SD. MAC: minimum alveolar anesthetic concentration. ${ }^{*} \mathrm{P}<0.05$ compared with the baseline value. ${ }^{\dagger} \mathrm{P}<0.05$ compared with and without inhaled anesthetics under hypercapnia. ${ }^{\ddagger} \mathrm{P}<0.05$ compared between normocapnia and hypercapnia with $1.0 \mathrm{MAC}$ of inhaled anesthetics.

in intracranial pressure. In our results, in both sevoflurane and desflurane groups, the rates of dilation in the arterioles and the venules under any conditions were similar. Both anesthetics might affect intracranial pressure via at least venous dilation. The cerebrovascular response to $\mathrm{CO}_{2}$ under anesthesia with sevoflurane or desflurane has been reported to be preserved in children, adults, and even in patients with previous stroke [8$10,14,18]$. The cerebrovascular response to $\mathrm{CO}_{2}$ is larger in patients anesthetized with isoflurane than in patients anesthetized with sevoflurane [10]. However, the precise effect of inhaled anesthetics on the vasodilatory response to hypercapnia was not established in the literature. In our results, 1.0 MAC of both sevoflurane or desflurane showed enhanced vasodilatory effects under hypercapnia, and the rate of vasodilation with desflurane was similar to that with sevoflurane. Therefore, it is suggested that the effect of desflurane on intracranial pressure is comparable to that of sevoflurane.

It has been reported that in patients undergoing craniotomy, the time from discontinuing administration of volatile anesthetics to extubation or consciousness was shorter in desflurane anesthesia than in sevoflurane anesthesia [3]. Desflurane might therefore be preferable for neurosurgery [3]. Vasodilation of the cerebral vasculature induces increments in intracranial pressure [7]. Both sevoflurane and desflurane can increase intracranial pressure through enhancement of the vasodilatory effect under hypercapnia. Therefore, it is important to maintain a normal $\mathrm{PaCO}_{2}$ level.

The limitation of the present study is that although a previous study demonstrated that pentobarbital has a limited effect on cerebrovascular tone [19], we cannot exclude the possibility that the pial vessel reactivity observed in our study was affected by the presence of pentobarbital.

In conclusion, both sevoflurane and desflurane induce similar cerebrovascular responses. Thus, desflurane can be used as safely as sevoflurane in neurosurgical anesthesia.

\section{Conflicts of Interest}

No potential conflict of interest relevant to this article was reported. 


\section{Author Contributions}

Koji Sakata (Data curation; Investigation; Writing - original draft)

Kazuhiro Kito (Methodology)

Naokazu Fukuoka (Methodology)

Kiyoshi Nagase (Writing - review \& editing)

Kumiko Tanabe (Writing - review \& editing)

Hiroki Iida (Supervision; Writing - review \& editing)

\section{ORCID}

Koji Sakata, https://orcid.org/0000-0001-8714-0329

Kazuhiro Kito, https://orcid.org/0000-0003-2388-7743

Naokazu Fukuoka, https://orcid.org/0000-0002-7932-4387

Kiyoshi Nagase, https://orcid.org/0000-0002-1039-167X

Kumiko Tanabe, https://orcid.org/0000-0002-3580-5701

Hiroki Iida, https://orcid.org/0000-0003-0924-598X

\section{References}

1. Brian JE Jr. Carbon dioxide and the cerebral circulation. Anesthesiology 1998; 88: 1365-86.

2. White PF, Tang J, Wender RH, Yumul R, Stokes OJ, Sloninsky A, et al. Desflurane versus sevoflurane for maintenance of outpatient anesthesia: the effect on early versus late recovery and perioperative coughing. Anesth Analg 2009; 109: 387-93.

3. Magni G, Rosa IL, Melillo G, Savio A, Rosa G. A comparison between sevoflurane and desflurane anesthesia in patients undergoing craniotomy for supratentorial intracranial surgery. Anesth Analg 2009; 109: 567-71.

4. Lenz C, Rebel A, van Ackern K, Kuschinsky W, Waschke KF. Local cerebral blood flow, local cerebral glucose utilization, and flowmetabolism coupling during sevoflurane versus isoflurane anesthesia in rats. Anesthesiology 1998; 89: 1480-8.

5. Lenz C, Frietsch T, Fütterer C, Rebel A, van Ackern K, Kuschinsky W, et al. Local coupling of cerebral blood flow to cerebral glucose metabolism during inhalational anesthesia in rats: desflurane versus isoflurane. Anesthesiology 1999; 91: 1720-3.

6. Matta BF, Heath KJ, Tipping K, Summors AC. Direct cerebral vasodilatory effects of sevoflurane and isoflurane. Anesthesiology 1999; 91: 677-80.

7. Sponheim S, Skraastad Ø, Helseth E, Due-Tønnesen B, Aamodt G, Breivik H. Effects of 0.5 and 1.0 MAC isoflurane, sevoflurane and desflurane on intracranial and cerebral perfusion pressures in children. Acta Anaesthesiol Scand 2003; 47: 932-8.

8. Nishiyama T, Matsukawa T, Yokoyama T, Hanaoka K. Cerebrovascular carbon dioxide reactivity during general anesthesia: a comparison between sevoflurane and isoflurane. Anesth Analg 1999; 89: 1437-41.

9. Rowney DA, Fairgrieve R, Bissonnette B. Cerebrovascular carbon dioxide reactivity in children anaesthetized with sevoflurane. Br J Anaesth 2002; 88: 357-61.

10. Kadoi Y, Saito S, Takahashi K. The comparative effects of sevoflurane versus isoflurane on cerebrovascular carbon dioxide reactivity in patients with previous stroke. J Anesth 2008; 22: 135-9.

11. Eger EI 2nd, Johnson BH. MAC of I-653 in rats, including a test of the effect of body temperature and anesthetic duration. Anesth Analg 1987; 66: 974-6.

12. Iida $\mathrm{H}$, Ohata $\mathrm{H}$, Iida $\mathrm{M}$, Watanabe $\mathrm{Y}$, Dohi S. Isoflurane and sevoflurane induce vasodilation of cerebral vessels via ATP-sensitive K+ channel activation. Anesthesiology 1998; 89: 954-60.

13. Crawford MW, Lerman J, Saldivia V, Carmichael FJ. Hemodynamic and organ blood flow responses to halothane and sevoflurane anesthesia during spontaneous ventilation. Anesth Analg 1992; 75: 1000-6.

14. Mielck F, Stephan H, Buhre W, Weyland A, Sonntag H. Effects of 1 MAC desflurane on cerebral metabolism, blood flow and carbon dioxide reactivity in humans. Br J Anaesth 1998; 81: 155-60.

15. Luginbuehl IA, Fredrickson MJ, Karsli C, Bissonnette B. Cerebral blood flow velocity in children anaesthetized with desflurane. Paediatr Anaesth 2003; 13: 496-500.

16. Johnston IH, Rowan JO. Raised intracranial pressure and cerebral blood flow. 3. Venous outflow tract pressures and vascular resistances in experimental intracranial hypertension. J Neurol Neurosurg Psychiatry 1974; 37: 392-402.

17. Nemoto EM. Dynamics of cerebral venous and intracranial pressures. Acta Neurochir Suppl 2006; 96: 435-7.

18. Luginbuehl IA, Karsli C, Bissonnette B. Cerebrovascular reactivity to carbon dioxide is preserved during hypocapnia in children anesthetized with 1.0 MAC, but not with 1.5 MAC desflurane. Can J Anaesth 2003; 50: 166-71.

19. Hatano Y, Nakamura K, Moriyama S, Mori K, Toda N. The contractile responses of isolated dog cerebral and extracerebral arteries to oxybarbiturates and thiobarbiturates. Anesthesiology 1989; 71: 80-6. 